\title{
Platelet-Rich Plasma Injections as a Treatment for Refractory Patellar Tendinosis: A Meta-Analysis of Randomised Trials
}

\author{
Leanne Dupley, MRCS ${ }^{1}$ and Charalambos P. Charalambous, FRCS ${ }^{1,2}$ \\ ${ }^{1}$ Department of Trauma and Orthopaedics, Blackpool Teaching Hospitals NHS Trust, Blackpool; ${ }^{2}$ School of Medicine, University of Central Lancashire, Preston, UK
}

\begin{abstract}
Purpose: Patellar tendinosis (PT) is a common condition amongst athletes. In this study, we perform a meta-analysis on randomised controlled trials (RCTs) to evaluate the use of platelet-rich plasma (PRP) for refractory PT.

Methods: A literature search was undertaken in various databases from their year of inception to October 2015. The primary outcome measure was the Victorian Institute of Sports Assessment-Patella (VISA-P) score.

Results: We identified 2 RCTs comparing PRP injections to alternative treatment options (extracorporeal shockwave therapy [ESWT] and dry needling of the tendon). Meta-analysis showed no significant difference in mean VISA-P scores between PRP injection and control at early assessment ( 2 or 3 months; estimated difference in means, 11.9; standard error [SE], 7.4; 95\% confidence interval [CI], -2.7 to 26.4; p=0.109). However, PRP was statistically better than control with regards to VISA-P scores at longer follow-up (6 months or longer; estimated difference in means, 12.7; SE, 4.4; 95\% CI, 4.1 to 21.3 ; $\mathrm{p}=0.004$ ).

Conclusions: There is a paucity of RCTs evaluating the role of PRP in PT. Our results suggest that, based on limited evidence, PRP is superior over other established non-surgical treatments (dry needling and ESWT) for refractory PT. Larger RCTs may allow better characterisation of the role of PRP in this condition.
\end{abstract}

Keywords: Patella, Tendinopathy, Platelet-rich plasma, Injection

\section{Introduction}

Patellar tendinosis (PT) is a clinical syndrome characterised by anterior knee pain and tenderness at the insertion of the patellar tendon on the inferior pole of the patella. Commonly known as “jumper's knee”, PT causes pain following repetitive running or jumping exercises, which usually settles with rest but returns when exercise is recommenced ${ }^{1)}$. A cross-sectional study by Lian

Received September 26, 2016; Revised December 30, 2016;

Accepted February 9, 2017

Correspondence to: Charalambos P. Charalambous, FRCS

Department of Trauma and Orthopaedics, Blackpool Teaching Hospitals NHS Trust, Whinney Heys Rd, Blackpool FY3 8NR, UK

Tel: +44-1253955983, Fax: +44-1253953530

E-mail: mr.charalambous@bfwhospitals.nhs.uk

This is an Open Access article distributed under the terms of the Creative Commons Attribution Non-Commercial License (http://creativecommons.org/licenses/by-nc/4.0/) which permits unrestricted non-commercial use, distribution, and reproduction in any medium, provided the original work is properly cited. et al. ${ }^{2)}$ showed that $22 \%$ of the 613 interviewed athletes either currently had symptoms of PT (14\%) or had previously experienced symptoms of PT (8\%). The prevalence of PT varied significantly between sports: volleyball and basketball showed the highest prevalence with $45 \%$ and $32 \%$ of participants affected, respectively; however, cycling had none, suggesting a relation to sports requiring high speed and power of the knee extensors.

PT can have a prolonged disease course, refractory to firstline non-surgical treatments (such as patellar tendon eccentric exercises), which can lead to many patients requiring additional intervention $^{3)}$. Platelet-rich plasma (PRP) injections have been shown to be beneficial to the healing and repair process of diseased or injured tendons, and have been used with promising results in tendinopathies such as epicondylitis ${ }^{4)}$ and Achilles tendinosis ${ }^{5)}$. Uncontrolled clinical studies have reported that PRP can improve symptoms in $\mathrm{PT}^{6,7}$; however, before the wide use of PRP can be recommended, high-quality controlled research evidence is required ${ }^{8,9)}$. Hence the aim of this study was to review the current literature with regards to randomised controlled trials (RCTs) 
that have compared PRP injections with other treatment options for PT and carry out a meta-analysis, pooling the results of such trials.

\section{Methods}

A search of PubMed, EMBASE, CINAHL (Cumulative Index to Nursing and Allied Health Literature), and Cochrane CENTRAL (Central Register of Controlled Trials) was conducted from their year of inception to October 2015 with the keywords: "patella" and "platelet", followed by a search with the keywords: "patellar" and "platelet". Broad search keywords were used, rather than specific terms, to ensure no articles were missed. There was no language limit and only fully published articles or abstracts were included. The Preferred Reporting Items for Systematic Review and Meta-Analysis ${ }^{10)}$ methodology guidance was employed. Full texts were reviewed for relevant articles or where a decision regarding inclusion could not be made from the title and abstract. The reference list of relevant articles was also examined for any additional articles not identified from the database search. Studies were included if they compared the use of PRP versus any other treatment for PT. Single case reports, reviews, and noncomparable studies were excluded. The methodology of the studies was carefully examined to ensure that they were true RCTs only. Data were extracted in a standardised manner. The common reported outcome for comparison was the Victorian Institute of Sports Assessment-Patella (VISA-P) score ${ }^{11)}$, which is the only validated scoring system developed specifically for patella tendinopathy. It assesses the severity of symptoms, patient's function, and ability to participate in sporting activities, giving a score from 0 (asymptomatic) to 100. The VISA-P takes into account patient's pain during activities such as squatting, lunging, sitting and the duration of time during training before the pain becomes activity limiting.

A meta-analysis of mean differences in VISA-P score was performed with use of a random-effects model. Summary mean differences, standard errors (SEs), and 95\% confidence intervals (CIs) were calculated. Heterogeneity was assessed using tau ${ }^{2}, I^{2}, Q$, and p-values. Data were analysed with CMA ver. 2 (Biostat Inc., Englewood, NJ, USA).

\section{Results}

From 556 published abstracts identified, 550 were excluded for non-pertinence including all non-RCTs. Six studies underwent a full abstract review, 3 of which were rejected due to not meeting inclusion criteria or duplication. Three full texts were reviewed; 1 was found to be a duplicate, which left 2 RCTs that met the inclusion criteria and hence were included in the meta-analysis (Fig. 1). The first RCT by Dragoo et al. ${ }^{12}$ in our meta-analysis compared leukocyte-rich PRP injections with ultrasound guided dry needling of the patella tendon in the treatment of PT. Patients in both the test and control groups were instructed to follow a program of eccentric exercises and had twice weekly physiotherapy sessions following the treatment.

The second included RCT by Vetrano et al. ${ }^{13)}$ compared leukocyte-rich PRP injections with focused extracorporeal shock wave therapy (ESWT). Both the PRP and ESWT groups were given a standardised stretching and muscle strengthening protocol to follow for 2 weeks.

Table 1 displays demographics and definitions of the two included studies. Both RCTs used the VISA-P questionnaire scores for reporting outcomes and this was the outcome utilised in the meta-analysis.

Meta-analysis of the two studies showed no significant differ-

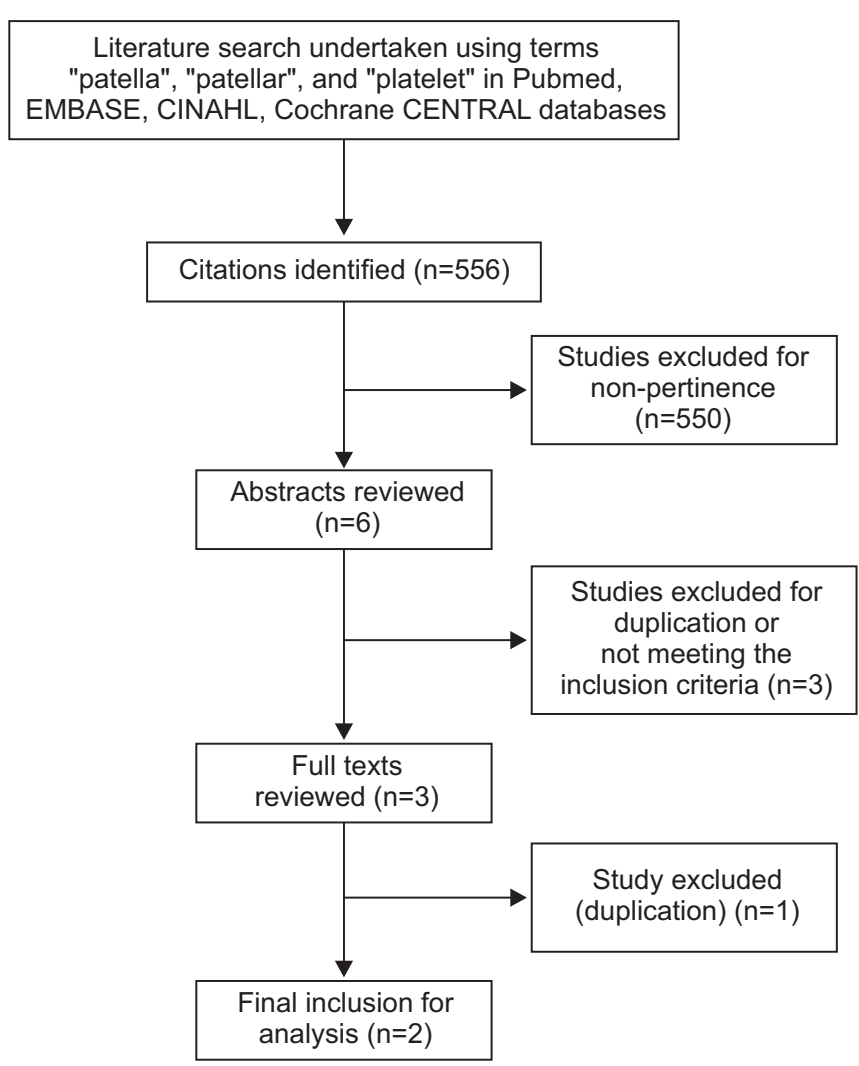

Fig. 1. Literature search and methodology of selection. CINAHL: Cumulative Index to Nursing and Allied Health Literature, CENTRAL: Central Register of Controlled Trials. 


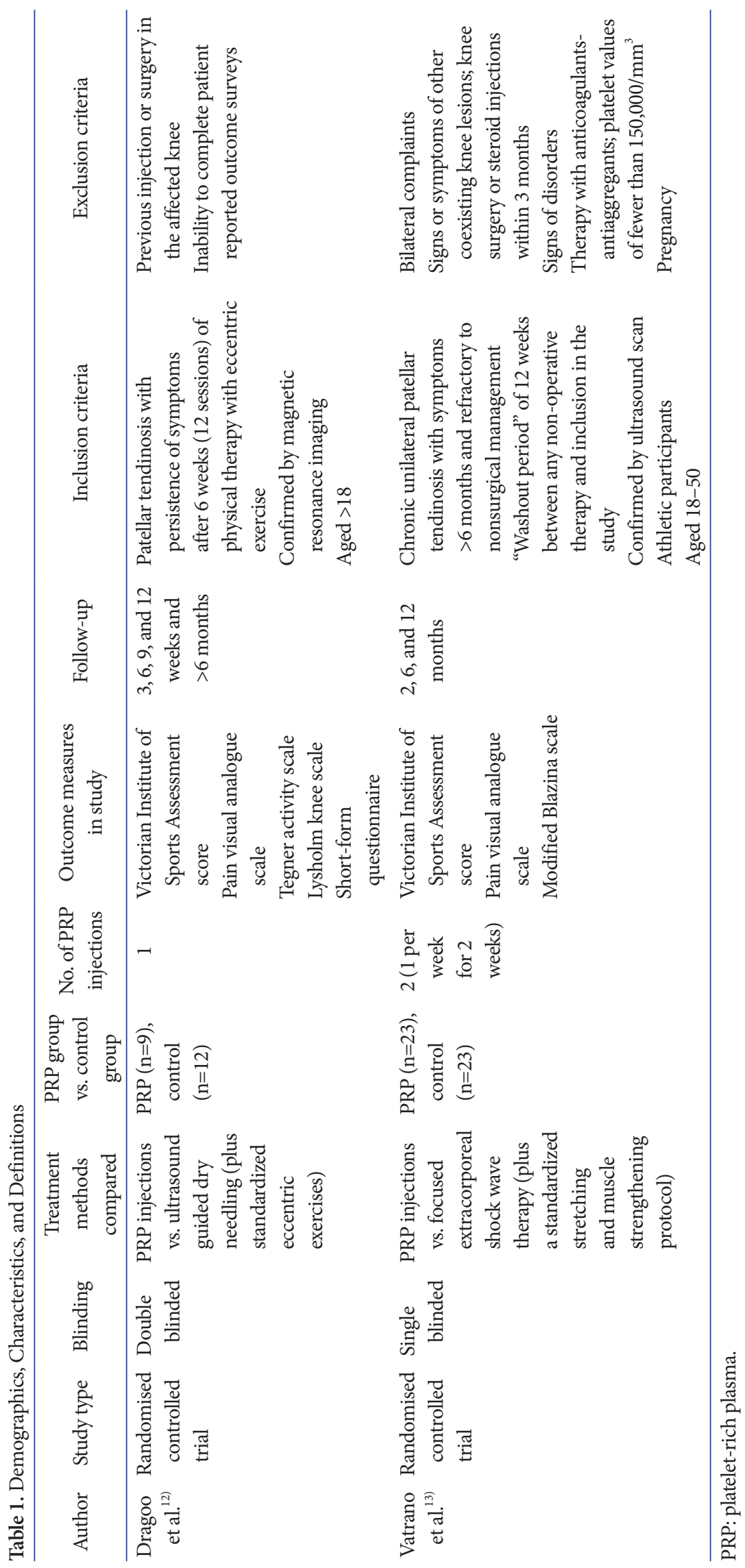




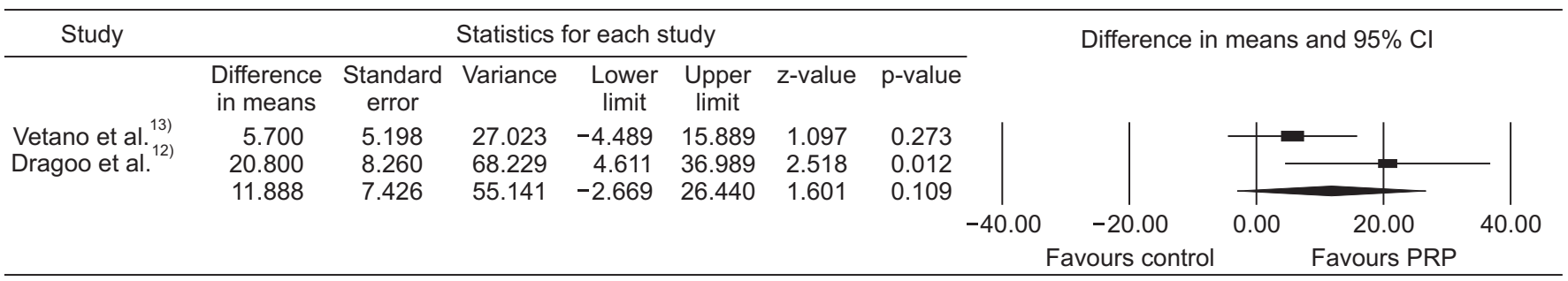

Meta-analysis

Fig. 2. Meta-analysis of short-term outcomes. CI: confidence interval, PRP: platelet-rich plasma.

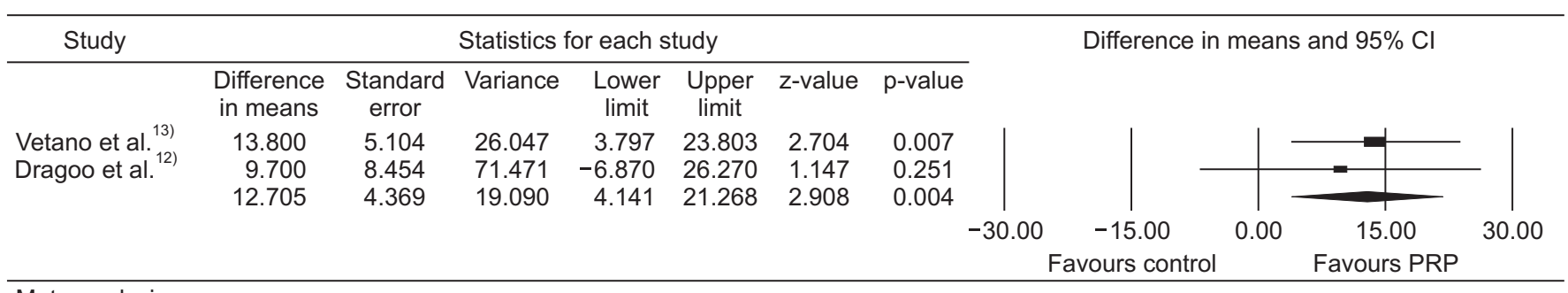

Meta-analysis

Fig. 3. Meta-analysis of long-term outcomes. CI: confidence interval, PRP: platelet-rich plasma.

ence in mean VISA-P scores between PRP injections and the control group at early assessment ( 2 or 3 months; difference in means, 11.9; $\mathrm{SE}, 7.4 ; 95 \% \mathrm{CI},-2.7$ to $26.4 ; \mathrm{p}=0.109$ ) (heterogeneity, $\operatorname{tau}^{2}=66.4 ; \mathrm{I}^{2}=58.2 \% ; \mathrm{Q}=2.39$; degrees of freedom, $1 ; \mathrm{p}=0.12$ ) (Fig. 2). However, PRP was statistically better than the control with regards to VISA-P scores at longer follow-up (at 6 months or more than 6 months; difference in means, 12.7 ; SE, $4.4 ; 95 \% \mathrm{CI}, 4.1$ to 21.3; $\mathrm{p}=0.004$ ) (heterogeneity, $\operatorname{tau}^{2}=0 ; \mathrm{I}^{2}=0 ; \mathrm{Q}=0.172$; degrees of freedom, $1 ; \mathrm{p}=0.68$ ) (Fig. 3).

\section{Discussion}

The most important finding in our meta-analysis is that PRP injections are statistically better than the control group (ESWT and dry needling) at longer term (6 months or more) follow-up suggesting that PRP is an effective and worthwhile treatment for PT.

PT is believed to be caused by degeneration of collagen fibres in the tendon, leading to micro-tearing, rather than due to an acute inflammatory process, which may be associated with failed healing due to poor vascularity within the tendon ${ }^{14)}$. Current treatments for PT include non-surgical options, such as eccentric exercises, ESWT, dry needling of the tendon, and PRP injections. These approaches are well established and they all aim to increase neovascularisation and promote cell growth and collagen synthesis. Chen et al. ${ }^{15)}$ showed that ESWT promoted healing of Achil- les tendonitis in rats by inducing transforming growth factor- $\beta 1$ (TGF- $\beta 1$ ) and insulin-like growth factor 1 (IGF-1) production. These factors are known to play important roles in mediating cell proliferation and tissue regeneration of tendons. Cell proliferation and collagen synthesis were also found to be increased in tendons following ESWT in a number of papers ${ }^{16,17}$. Further studies have shown that ESWT can cause neovascularisation at the bonetendon junction due to release of certain angiogenic mediators, such as vascular endothelial growth factor (VEGF), endothelial nitric oxide synthase, and proliferating cell nuclear antigen ${ }^{18,19)}$. Indeed a number of studies have found ESWT to be beneficial to patients with PT over physiotherapy alone $\mathrm{e}^{20,21)}$.

Dry needling of affected tendons is thought to work in a similar way by causing bleeding and inflammation with local release of beneficial growth factors ${ }^{22,23)}$.

An RCT in 2006 by Bahr et al. ${ }^{24)}$ showed no benefit of surgical tenotomy over eccentric exercises alone for the treatment of PT. Further studies by Peers et al. ${ }^{25)}$ showed comparable results between ESWT and surgical intervention in a cross-sectional outcome analysis for chronic patella tendinosis. Many centres now reserve surgical tenotomy as the last treatment option for PT due to inconsistent results and potential morbidity associated with surgery ${ }^{24,26)}$.

PRP injections are also not a new concept and have been used since the 1970s. There are various PRP kits available; however, the common process involves taking a venous sample of blood 
from the patient using a venepuncture device and a bottle containing an anticoagulant to prevent platelet activation prior to its use. This sample is placed into a centrifuge which is spun resulting in separation of the whole blood contents into three layers: the top platelet rich layer containing some white blood cells (WBC), the intermediate "buffy" layer containing mostly WBCs, and the third layer rich in red blood cells. The top platelet-rich layer can be removed and used as leukocyte-rich PRP injections. A further centrifugation of the top layer and "buffy" layer can be done to produce a pure PRP injection ${ }^{27}$. Both studies in our meta-analysis used a leukocyte-rich PRP injection.

Platelets have the potential to release growth factors, such as TGF- $\beta$, VEGF, platelet-derived growth factor, IGF-1, and fibroblast growth factor, as well as cytokines which mediate healing within tendons ${ }^{7,28,29)}$. There have been several non-controlled studies reporting good outcomes with PRP use in PT. In a 31 patient study, Filardo et al. ${ }^{7)}$ found a statistically significant improvement in sport activity levels at the end of a course of PRP injections in patients with chronic refractory PT compared to those treated with physiotherapy alone. In a prospective study by Kon et al. ${ }^{6}, 20$ male athletes with refractory PT underwent PRP injections and all had a statistical improvement in questionnaire scores at 6-month follow-up.

However, as our study demonstrates there is a paucity in RCTs evaluating the role of PRP injections in PT. We were able to identify only 2 such studies and these differed in the PRP regime they administered and in the control group they utilised. Vetrano et al. ${ }^{13)}$ found that both PRP injections and ESWT improved the VISA-P score at 2, 6, and $>12$ months ( $\mathrm{p}<0.005$ for all). There was no significant difference in the scores at baseline and 2 months; however, there was significant difference between the groups at 6 months (PRP injection group, $86.7 \pm 14.2$ vs. ESWT group, $73.7 \pm 19.9 ; \mathrm{p}=0.014)$ and 12 months of follow-up (91.3 \pm 9.9 vs. 77.6 $\pm 19.9 ; \mathrm{p}=0.026)$. Dragoo et al. ${ }^{12}$ found the VISA-P scores of the PRP group had improved significantly more than the dry needling group at 12 weeks $(\mathrm{p}=0.02)$; however, the difference between the two groups was not significant at $>26$ weeks $(\mathrm{p}=0.66)$.

The visual analog scale (VAS) score for pain was also used as an outcome measure in both included studies; however, this is not a specific questionnaire for PT. Vetrano et al. ${ }^{13)}$ showed that, similarly to the VISA-P score, there was improvement in the VAS scores for 2 months, albeit not significant $(\mathrm{p}=3.58)$, but there was significant difference between the VAS scores at 6 months (PRP injection group, $2.4 \pm 1.9$ vs. ESWT group, $3.9 \pm 2.3 ; \mathrm{p}=0.028$ ) and 12 months ( $1.5 \pm 1.7$ vs $3.2 \pm 2.4 ; \mathrm{p}=0.009$ ). In the Dragoo et al. ${ }^{12)}$ study, the VAS pain score improved for both the dry needling and PRP groups at 12 and > 26 weeks; however, there was no sig- nificant difference between the two groups at either follow-up (12 weeks, $\mathrm{p}=0.13$; $>26$ weeks, $\mathrm{p}=0.96$ ).

It is of interest that Dragoo et al. ${ }^{12)}$ found significant early (12 week) improvements in scores between the PRP and dry needling groups, but no significant differences at the late term follow-up (>26 weeks). In contrast, Vetrano et al. ${ }^{13)}$ found no significant difference between PRP and ESWT at early (2 month) followup, but the PRP group scored significantly better on VISA-P and VAS at 6 and 12 months. One possible explanation for these contrasting findings could be the number of PRP injections given: the Dragoo et al. ${ }^{12)}$ PRP group received one PRP injection, whereas in the study by Vetrano et al. ${ }^{13}$, the PRP group received two injections two weeks apart. It is possible that two PRP injections lead to an increased and prolonged inflammatory response, which takes a longer time to settle and allow clinical improvement.

When the results of the 2 studies were combined through a meta-analysis, there was no significant difference in mean VISA$\mathrm{P}$ scores between PRP injection and control at early assessment (2 or 3 months) but PRP was statistically better than control with regards to VISA-P scores at longer follow-up (at 6 months or more than 6 months), suggesting that PRP is a viable alternative to other non-surgical options.

The main limitation of this meta-analysis is that there are only 2 trials included, both of which were small in size and showed substantial heterogeneity with regards to the regime of PRP injections and type of control group used. The follow-up in our study was limited to 6 months or " 6 months or more", and it would be beneficial to be able to compare outcomes further down the line with a follow-up at 12 months, etc. Nevertheless, meta-analyses are useful even in the presence of a small number of studies in that they allow pooling of data and potentially an early identification of a beneficial or otherwise effect, whilst larger trials are awaited.

It is also possible that the difference at 6 months between PRP and control maybe due to some other factors other than the PRP injection itself. However, we feel this is less likely to be the cause of the observed difference, as patients were randomly assigned to the treatment and control groups, which would help deal with any unknown confounders. In addition, both groups received similar treatments other than the PRP injection-i.e. the same course of physiotherapy/exercise regime to follow.

Hence, within the limitations of this study, our results suggest that PRP does seem to be superior for refractory PT over other established non-surgical treatments (such as dry-needling and ESWT) at 6 months post treatment. Larger RCTs comparing $\mathrm{PRP}$ versus other established treatments in PT are needed. In the 
meantime, however, based on our results we would recommend the use of PRP in the treatment of PT.

\section{Conflict of Interest}

No potential conflict of interest relevant to this article was reported.

\section{References}

1. Ferretti A, Puddu G, Mariani PP, Neri M. The natural history of jumper's knee: patellar or quadriceps tendonitis. Int Orthop. 1985;8:239-42.

2. Lian OB, Engebretsen L, Bahr R. Prevalence of jumper's knee among elite athletes from different sports: a cross-sectional study. Am J Sports Med. 2005;33:561-7.

3. Woodley BL, Newsham-West RJ, Baxter GD. Chronic tendinopathy: effectiveness of eccentric exercise. Br J Sports Med. 2007;41:188-98.

4. Halpern B, Chaudhury S, Nguyen J, Hayter C, Hough L. Clinical and magnetic resonance imaging outcomes following platelet rich plasma treatment for elbow tendinosis. J Arthritis. 2015;4:182.

5. Filardo G, Kon E, Di Matteo B, Di Martino A, Tesei G, Pelotti P, Cenacchi A, Marcacci M. Platelet-rich plasma injections for the treatment of refractory Achilles tendinopathy: results at 4 years. Blood Transfus. 2014;12:533-40.

6. Kon E, Filardo G, Delcogliano M, Presti ML, Russo A, Bondi A, Di Martino A, Cenacchi A, Fornasari PM, Marcacci M. Platelet-rich plasma: new clinical application: a pilot study for treatment of jumper's knee. Injury. 2009;40:598-603.

7. Filardo G, Kon E, Della Villa S, Vincentelli F, Fornasari PM, Marcacci M. Use of platelet-rich plasma for the treatment of refractory jumper's knee. Int Orthop. 2010;34:909-15.

8. de Almeida AM, Demange MK, Sobrado MF, Rodrigues MB, Pedrinelli A, Hernandez AJ. Patellar tendon healing with platelet-rich plasma: a prospective randomized controlled trial. Am J Sports Med. 2012;40:1282-8.

9. Lyras DN, Kazakos K, Verettas D, Botaitis S, Agrogiannis G, Kokka A, Pitiakoudis M, Kotzakaris A. The effect of plateletrich plasma gel in the early phase of patellar tendon healing. Arch Orthop Trauma Surg. 2009;129:1577-82.

10. Moher D, Liberati A, Tetzlaff J, Altman DG; PRISMA Group. Preferred reporting items for systematic reviews and metaanalyses: the PRISMA statement. Int J Surg. 2010;8:336-41.

11. Visentini PJ, Khan KM, Cook JL, Kiss ZS, Harcourt PR,
Wark JD. The VISA score: an index of severity of symptoms in patients with jumper's knee (patellar tendinosis): Victorian Institute of Sport Tendon Study Group. J Sci Med Sport. 1998;1:22-8.

12. Dragoo JL, Wasterlain AS, Braun HJ, Nead KT. Platelet-rich plasma as a treatment for patellar tendinopathy: a doubleblind, randomized controlled trial. Am J Sports Med. 2014; 42:610-8.

13. Vetrano M, Castorina A, Vulpiani MC, Baldini R, Pavan A, Ferretti A. Platelet-rich plasma versus focused shock waves in the treatment of jumper's knee in athletes. Am J Sports Med. 2013;41:795-803.

14. Khan KM, Bonar F, Desmond PM, Cook JL, Young DA, Visentini PJ, Fehrmann MW, Kiss ZS, O'Brien PA, Harcourt PR, Dowling RJ, O'Sullivan RM, Crichton KJ, Tress BM, Wark JD. Patellar tendinosis (jumper's knee): findings at histopathologic examination, US, and MR imaging: Victorian Institute of Sport Tendon Study Group. Radiology. 1996; 200:821-7.

15. Chen YJ, Wang CJ, Yang KD, Kuo YR, Huang HC, Huang YT, Sun YC, Wang FS. Extracorporeal shock waves promote healing of collagenase-induced Achilles tendinitis and increase TGF-betal and IGF-I expression. J Orthop Res. 2004; 22:854-61.

16. Berta L, Fazzari A, Ficco AM, Enrica PM, Catalano MG, Frairia R. Extracorporeal shock waves enhance normal fibroblast proliferation in vitro and activate mRNA expression for TGF-beta1 and for collagen types I and III. Acta Orthop. 2009;80:612-7.

17. Vetrano M, d'Alessandro F, Torrisi MR, Ferretti A, Vulpiani MC, Visco V. Extracorporeal shock wave therapy promotes cell proliferation and collagen synthesis of primary cultured human tenocytes. Knee Surg Sports Traumatol Arthrosc. 2011;19:2159-68.

18. Wang CJ, Wang FS, Yang KD, Weng LH, Hsu CC, Huang CS, Yang LC. Shock wave therapy induces neovascularization at the tendon-bone junction: a study in rabbits. J Orthop Res. 2003;21:984-9.

19. Wang CJ, Huang HY, Pai CH. Shock wave-enhanced neovascularization at the tendon-bone junction: an experiment in dogs. J Foot Ankle Surg. 2002;41:16-22.

20. Vulpiani MC, Vetrano M, Savoia V, Di Pangrazio E, Trischitta D, Ferretti A. Jumper's knee treatment with extracorporeal shock wave therapy: a long-term follow-up observational study. J Sports Med Phys Fitness. 2007;47:323-8.

21. Wang CJ, Ko JY, Chan YS, Weng LH, Hsu SL. Extracorporeal 
shockwave for chronic patellar tendinopathy. Am J Sports Med. 2007;35:972-8.

22. Housner JA, Jacobson JA, Morag Y, Pujalte GG, Northway RM, Boon TA. Should ultrasound-guided needle fenestration be considered as a treatment option for recalcitrant patellar tendinopathy? A retrospective study of 47 cases. Clin J Sport Med. 2010;20:488-90.

23. Nagraba L, Tuchalska J, Mitek T, Stolarczyk A, Deszczynski J. Dry needling as a method of tendinopathy treatment. Ortop Traumatol Rehabil. 2013;15:109-16.

24. Bahr R, Fossan B, Loken S, Engebretsen L. Surgical treatment compared with eccentric training for patellar tendinopathy (jumper's knee): a randomized, controlled trial. J Bone Joint Surg Am. 2006;88:1689-98.

25. Peers KH, Lysens RJ, Brys P, Bellemans J. Cross-sectional outcome analysis of athletes with chronic patellar tendinopathy treated surgically and by extracorporeal shock wave therapy. Clin J Sport Med. 2003;13:79-83.

26. Andres BM, Murrell GA. Treatment of tendinopathy: what works, what does not, and what is on the horizon. Clin Orthop Relat Res. 2008;466:1539-54.

27. Dhurat R, Sukesh M. Principles and methods of preparation of platelet-rich plasma: a review and author's perspective. J Cutan Aesthet Surg. 2014;7:189-97.

28. Kim ES, Park EJ, Choung PH. Platelet concentration and its effect on bone formation in calvarial defects: an experimental study in rabbits. J Prosthet Dent. 2001;86:428-33.

29. Sanchez AR, Sheridan PJ, Kupp LI. Is platelet-rich plasma the perfect enhancement factor? A current review. Int J Oral Maxillofac Implants. 2003;18:93-103. 